\section{Announcing New Article Categories}

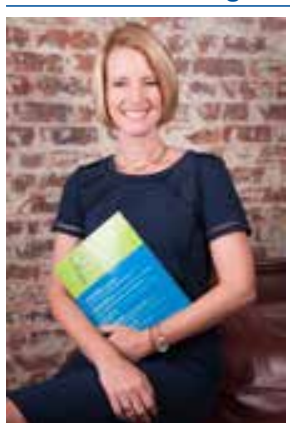

As the calendar turns to January, we often look at the coming year with fresh perspectives, sometimes resolving to make a change or accomplish a goal. So, the New Year is the perfect time to announce a refresh to the article categories for the Journal of Managed Care E Specialty Pharmacy (JMCP). We hope that you will find these categories to be clear, relevant, and specific and that they encompass the types of articles that will address the big issues in managed care and specialty pharmacy.

The overwhelming majority of articles that JMCP publishes will continue to fall in the Research category, since our mission is to advance the use of the scientific method in managed care pharmacy. We will continue to encourage submissions in all therapeutic categories, clinical management, formulary management, and specialty pharmacy.

Because the new article categories are more specific, including word and table/figure limits, we will take a phased approach to implementation. Beginning January 1, 2016, the new article categories will replace the old categories in our online submission system. However, we will not enforce the word limit requirements until March 1, 2016. More details on each category can be found in the Instructions for Authors at http://amcp.org/JMCP_AuthorGuidelines/.

I would like to personally thank each of you for your contributions to JMCP, either as a reader, peer reviewer, author, or all three! Because of your interest, JMCP has grown rapidlyincreasing from 9 to 12 issues per year in 2014 and publishing a record number of articles since then. We look forward to continuing to connect authors with readers in order to advance the profession of managed care pharmacy.

Cheers to a happy 2016 !

Laura E. Happe, PharmD, MPH

Editor-in-Chief

lhappe@jmcp.org

\section{New Article Categories}

Research. These articles report experimental or observational studies that use the scientific method. Research articles should not exceed 4,000 words in the body of the manuscript and should have a maximum of 5 tables and/or figures. These articles should be accompanied by a structured abstract of no more than 450 words and can include up to 2 appendices.
Research articles should follow reporting standards based on the design of the study:

- CONSORT for clinical trials

- $\underline{\text { STROBE for observational studies }}$

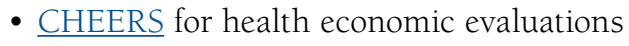

- GRACE for observational studies of comparative effectiveness

- ISPOR Good Research Practices for Comparative

Effectiveness Research for observational studies of comparative effectiveness

Research Brief. These articles are similar to Research; however, this category is reserved for small or pilot studies that generally have limited generalizability or descriptive studies that may not test a hypothesis or have comparative study groups. Research Briefs should not exceed 2,500 words and should have a maximum of 2 tables and/or figures. These articles should be accompanied by a structured abstract of no more than 350 words.

Systematic Review. These articles report on literature reviews conducted using the scientific method. Systematic reviews should include a guiding hypothesis or question, criteria to determine study inclusion, extraction and analysis of results, and conclusions based on the presence or absence of evidence identified in the literature. This article category includes meta-analyses and systematic literature reviews and should be reported using the PRISMA Statement. Systematic reviews should have a structured abstract of no more than 450 words, not exceed 4,000 words in the body of the manuscript, and have a maximum of 5 tables and/or figures with no more than 2 appendices.

Best Practices. These articles, or case studies, report the experience of innovative programs aimed at improving evolving challenges in the practice of managed care pharmacy. Articles should include a thorough program description, observations, and implications. Editorial priorities are for programs that address a timely topic or for other reasons that do not lend themselves to evaluation using the scientific method. Best Practices should contain a structured abstract of no more than 350 words, not exceed 2,500 words in the body of the manuscript, and have a maximum of 2 tables and/or figures.

Viewpoints. These articles are timely topical reviews that are relevant to JMCP's readership. Articles should be well referenced, be presented in a clear and scholarly manner, and address clinical, economic, policy, and patient perspectives. 
Viewpoints should contain an unstructured abstract of no more than 350 words, not exceed 2,500 words in the body of the manuscript, and have a maximum of 2 tables and/or figures. Viewpoints may be unsolicited or solicited by the editors.

Letters to the Editor. Letters to the Editor that discuss a timely topic relevant to managed care pharmacy or a recently published study are considered for publication. Submitted letters should not exceed 500 words in the body and 5 references. Letters in response to a recently published study should be submitted within 4 weeks of the publication of the original article. Letters to the Editor are not peer reviewed and are published at the discretion of the editor.
AMCP Meeting Proceedings. As the peer-reviewed journal of the Academy of Managed Care Pharmacy, JMCP accepts submissions from AMCP reporting the proceedings of association meetings that are timely and relevant to JMCP's readership. As with all articles, AMCP Meeting Proceedings are peer reviewed, with a special emphasis on ensuring that the content is presented in an unbiased manner, is a factual representation of what occurred at the meeting, and contains appropriate attributions. AMCP Meeting Proceedings should have an unstructured abstract of no more than 450 words, not exceed 3,000 words in the body of the manuscript, and have a maximum of 5 tables and/or figures. As with all reviewed articles, the final publication decision is made by JMCP editors.

\section{JOURNAL OF MANAGED CARE \& SPECIALTY Please join PHARMACY JMCP peer reviewers by completing a reviewer profile at jmcp.msubmit.net \\ If you have questions, please call or e-mail Jennifer Booker, Managing Editor e-mail: jmcpreview@amcp.org tel.: 703.317.0725
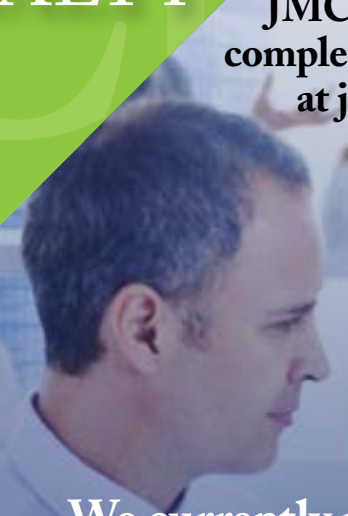 \\ We currently need expert reviewers in: \\ - Behavioral Health - Cardiovascular Disease - Diabetes \\ - Formulary Management $\cdot$ Hepatitis C $\bullet$ Multiple Sclerosis \\ - Oncology $\bullet$ Outcomes-based Contracting - Research Design - Specialty Drug Management}

